\title{
Competencias transversales en la formación de especialistas en pediatría, Universidad de Chile
}

\author{
L. Schonhaut-Berman ${ }^{a}$, T. Millán-Klusse ${ }^{b}$, C. Hanne-Altermattc
}

Introducción. El Programa de Formación de Especialistas en Pediatría (PFEP) debe responder a las necesidades futuras de la salud, políticas del país y, además, modernizarse acorde con las evidencias de la educación en ciencias de la salud, las que recomiendan la educación basada en competencias. Objetivo. El objetivo del estudio fue identificar las competencias transversales (CT) que debe lograr el licenciado del PFEP de la Universidad de Chile y evaluar su adquisición. Sujetos y métodos. Estudio cualitativo, descriptivo. Entre noviembre de 2006 y enero de 2007 se seleccionaron informantes clave mediante entrevistas en profundidad: ocho líderes, seis residentes y seis licenciados del PFEP de la Universidad de Chile, y se les preguntó por la relevancia y el logro de seis CT. Resultados. Para enfrentar los actuales desafíos laborales y epidemiológicos, se consideró fundamental la capacitación como médico de cabecera en pediatría ambulatoria y clínicas de continuidad, la adquisición de competencias de urgencia pediátrica y el manejo básico del niño hospitalizado. Se dio relevancia a la incorporación de CT carentes en el PFEP, como bioética, relación médico-paciente-familia, salud pública, trabajo en equipo y medicina basada en la evidencia (MBE). La docencia fue considerada como una actividad inherente al ejercicio médico, y se pudo ofrecer como un electivo al igual que la gestión. Conclusión. La adquisición de CT, más allá de las tradicionales competencias clínicas, es un desafío para la actual formación del pediatra y su adecuada preparación para el ejercicio profesional.

Palabras clave. Chile. Competencias transversales. Pediatría ambulatoria. Programa de formación de especialistas en pediatría. Residentes de pediatría. Transversal competencies for specialists training in
the Pediatrics Residency Program, University of Chile

Introduction. Teaching guidelines and the pediatrics exercise must adequate to the health requirements of the population needs and country politics, taking in consideration the new tendencies in medical education, with the introduction of competencies in the specialists training programs. Aim. To identify the competencies transversal to all specialties training programs (CT), that are important for the Pediatrics Residency Program (PFEP) at the Chile University. Subjects and methods. Qualitative and interpretive study, performed between November 2006 and January 2007. Key persons were interviewed (6 residents, 6 graduates and 8 opinion leaders) about the relevance and achievement of six CT. Results. The interviewed highlighted the necessity of trained pediatricians in ambulatory settings and continuity clinics, the acquisition of competencies in emergency units and basic skills in hospital training. They considered that the current training is lacking of important CT such as bioethics, physician-patient and family communication, public health, team work and evidence based medicine. Teaching skills and practice management are part of the medical profession; they could be introduced as an elective activity. Conclusion. The acquisition of CT during the PFEP transcend beyond the traditional clinical competencies, it is a challenge to introduce them in the PFEP.

Key words. Ambulatory pediatrics. Chile. Pediatrics Residency Program. Residents. Transversal competencies.

\section{Introducción}

En el contexto de la movilidad propia de la era de la globalización, de la transición epidemiológica y demográfica, las transformaciones sociales, el

\footnotetext{
Departamento de Pediatría Norte.

b Departamento de Pediatría Occidente Escuela de Medicina. Facultad de Medicina. Universidad de Chile. Independencia, Chile.

Correspondencia:

Dra. Luisa Schonhaut B. Departamento de Pediatría Norte. Facultad de Medicina. Universidad de Chile. Profesor Zañartu, 1085. 70005 Independencia, Chile.

E-mail Ischonhaut@med.uchile.cl
} 
impulso de la informática y la mayor complejidad científico-tecnológica, sumado a un mercado laboral cambiante [1,2], el profesional debe desplegar un abanico de herramientas que van más allá de los conocimientos teóricos, para desenvolverse en las distintas situaciones laborales y enfrentar los imprevistos.

Desde el informe Flexner, formulado hace casi un siglo para el pregrado de medicina, la educación basada en competencias se ha convertido en la mayor revolución en la educación en ciencias de la salud [3]. Este enfoque pone énfasis en el producto final, por lo que permitiría priorizar y adecuar los objetivos del aprendizaje en todos los niveles de la formación.

Se entiende por competencias la 'combinación dinámica de atributos -con respecto al conocimiento y su aplicación, a las actitudes y a las responsabilidades- que describen los resultados del aprendizaje de un determinado programa' [4].

Las competencias pueden ser agrupadas según si son específicas o genéricas. Las específicas son aquéllas que se asocian a áreas de conocimiento concretas, caracterizan una profesión y la distinguen de otras; las genéricas son comunes a todas las disciplinas, permiten a los profesionales adaptarse a nuevas situaciones, mantenerse actualizados y superar problemas laborales [5].

En el último grupo podríamos incluir un conjunto de competencias que son transversales a las carreras de salud y especialidades médicas. La priorización de dichas competencias no es sencilla y puede determinarse a partir de métodos diferentes [6], siendo aceptadas la opinión de expertos y también metodologías cualitativas, como la triangulación de percepciones de distintos actores [7].

El Accreditation Council for Graduate Medical Education (ACGME) fija los dominios de competencias que deben adquirir los residentes de los programas de formación de especialistas, que se han integrado progresivamente a los programas existentes [8].

Si se sigue la tendencia de innovación curricular, en 1998, la Universidad de Chile implementó un currículo basado en competencias para la formación de pregrado [9], pero no así para el posgrado, que continúa desarrollándose con un enfoque asistencial y flexneriano [10].

El Programa de Formación de Especialistas en Pediatría (PFEP) de la Universidad de Chile se desarrolla bajo el alero de cinco centros formadores ubicados en los servicios de pediatría de los

\section{Tabla I. Pauta de las entrevistas.}

$¿$ Cree usted que las competencias actuales de los licenciados del PFEP de la Universidad de Chile son acordes con las necesidades del país?

¿Cuáles son, a su juicio, las competencias que un licenciado debe tener en cuanto a:

- competencias clínicas generales?

- competencias en ética y humanística?

- competencias en comunicación médico-paciente?

- competencias en gestión: dirección de programas, dirección de establecimientos?

- competencias en análisis crítico de datos, medicina basada en evidencias?

- competencias en docencia?

- algún otro aspecto que desearía agregar?

hospitales de la Región Metropolitana. Pese a los esfuerzos que se han realizado a lo largo de los últimos años por unificar dichos programas, los matices y las actividades que se desarrollan son diferentes. En todos ellos el enfoque del PFEP es primordialmente clínico, de alta complejidad intrahospitalaria [11], con una rotación por pediatría ambulatoria variable entre las distintas especialidades clínicas, pero que no sobrepasa en ninguno el $10 \%$ del total del programa [12].

Si se consideran la realidad epidemiológica y laboral y, además, las tendencias en educación en salud, surgen una serie de preguntas: ¿será necesaria la incorporación de competencias transversales (CT), además de las tradicionales competencias clínicas, en los PFEP? ¿Qué otras capacidades requieren los pediatras para su desempeño profesional en el mundo actual?

El objetivo de este estudio, que forma parte de una línea de investigación sobre las competencias que deben lograr los residentes de pediatría [10], fue identificar la relevancia y adquisición de las CT en el PFEP de la Universidad de Chile.

\section{Sujetos y métodos}

Entre los meses de noviembre de 2006 y enero de 2007 se realizó un estudio cualitativo y des- 
criptivo a través de entrevistas en profundidad, realizadas por un mismo entrevistador, de forma confidencial, anónima y con consentimiento informado, a un total de 20 informantes clave distribuidos en: seis residentes $(\mathrm{R})$ y seis licenciados (P) del PFEP de la Universidad de Chile, y además a ocho líderes (E) en pediatría (profesores encargados del Programa, directores de departamentos de pediatría, jefes de servicio público y privado y el presidente de la Organización Científica Pediátrica). Para las entrevistas se diseñó y aplicó una pauta guía (Tabla I).

Dado que en los estudios cualitativos no hay reglas para el tamaño de la muestra, ya que depende de lo que será útil para el propósito de la investigación, se utilizó el muestreo intencional propio del método cualitativo, cuya lógica es seleccionar 'casos ricos en información' para estudiarlos en profundidad [13]. Se buscó que estuvieran representados residentes y licenciados de los distintos hospitales universitarios en los que se desarrolla el PFEP, se determinó su perfil en cuanto a género, experiencia laboral previa y actividad laboral posterior. Se identificaron líderes con cargos relevantes en distintos ámbitos de la pediatría.

Para determinar el número total de sujetos de la muestra se utilizó el criterio de saturación o redundancia, el cual se fundamenta en una continua interpelación entre la recogida y el análisis sistemático de datos, desarrollado durante la investigación; una vez que se construyen los significados que los participantes ven como su realidad social se deja de preguntar [14].

A través del método de la comparación constante, el investigador codifica y analiza los datos de forma simultánea para desarrollar conceptos. Su aplicación supone un contraste de las categorías, propiedades e hipótesis que surgen a lo largo del estudio en sucesivos contextos.

Basado en la definición de competencias del Comité Central del Instituto para la Educación Médica Internacional 2003 [15] y la Escuela de Medicina de la Universidad de Chile $[9,16,17]$, se seleccionaron cinco CT por ser consideradas las más relevantes, además de las competencias clínicas.

Las CT por las que se indagó son: comunicación efectiva en la relación médico-paciente, bioética, docencia, gestión y medicina basada en la evidencia (MBE). Se preguntó por la relevancia atribuida y por la formación durante el PFEP de cada una de las CT estudiadas.
El análisis de la información se realizó de acuerdo a las recomendaciones de la investigación cualitativa [18], que incluyeron las etapas siguientes:

- Trascripción de las entrevistas.

- Lectura fluctuante de las entrevistas.

- Comparación de los datos.

- Análisis de contenido de los datos.

- Reducción de la información.

- Integración de categorías y sus propiedades con disposición de datos en matrices.

- Delimitación de la teoría que se plantea.

- Validación por el equipo de investigación.

- Redacción de la teoría, tras un proceso de saturación de los incidentes pertenecientes a cada categoría, siguiendo el esquema de las dimensiones por las cuales se indagó.

Con la finalidad de garantizar el rigor científico se utilizó la confirmación como estrategia de credibilidad que permite seguridad de que los resultados de la investigación no están sesgados. Para ello la información se devolvió a un grupo de cinco expertos (uno de cada especialidad clínico, tres de los cuales habían sido incluidos en el grupo de entrevistados), quienes confirmaron la información obtenida y la enriquecieron con sus comentarios.

\section{Resultados}

En la tabla II se muestra la matriz de análisis comparativo entre las respuestas de los tres grupos entrevistados en cuanto a las principales competencias que se deberían adquirir en el PFEP. A continuación, se agrupan, enumeran y resumen las respuestas obtenidas.

\section{Competencias clínicas generales}

Los entrevistados consideraron fundamental lograr una capacidad resolutiva en los distintos escenarios en que atienden al paciente, especialmente en la atención ambulatoria y de urgencia. Los licenciados profundizaron en esto, y enfatizaron la importancia de lograr una atención integral, con enfoque biopsicosocial, aspecto que los líderes denominaron pediatría del desarrollo. Hubo coincidencia en que estas competencias son deficitarias en el PFEP. 
Tabla II. Análisis comparativo de la relevancia de las competencias transversales entre residentes, licenciados y líderes: coincidencias, énfasis y particularidades.

\begin{tabular}{|c|c|c|c|}
\hline Competencias & Residentes & Licenciados & Líderes \\
\hline $\begin{array}{l}\text { Clínicas } \\
\text { generales }\end{array}$ & $\begin{array}{l}\text { Pediatría ambulatoria (5/6) } \\
\text { Diagnóstico }(2 / 6) \\
\text { Capacidad resolutiva (4/6) } \\
\text { Manejo de la patología } \\
\text { prevalente }(2 / 6) \\
\text { Derivación adecuada }(2 / 6) \\
\text { Manejo intrahospitalario } \\
\text { básico }(1 / 6)\end{array}$ & $\begin{array}{l}\text { Pediatría ambulatoria, integral } \\
\text { con enfoque biopsicosocial: } \\
\text { 'la nueva pediatría' }(6 / 6) \\
\text { Diagnóstico }(3 / 6) \\
\text { Capacidad resolutiva }(3 / 6) \\
\text { Manejo de la patología } \\
\text { prevalente }(3 / 6) \\
\text { Derivación adecuada }(2 / 6) \\
\text { Manejo intrahospitalario } \\
\text { básico }(1 / 6)\end{array}$ & $\begin{array}{l}\text { Pediatría ambulatoria, integral } \\
\text { con enfoque biopsicosocial, } \\
\text { pediatría del desarrollo } \\
\text { o'la nueva pediatría' }(8 / 8) \\
\text { Papel del pediatra como } \\
\text { médico de cabecera }(3 / 8) \\
\text { Capacidad resolutiva }(5 / 8) \\
\text { Manejo de la patología } \\
\text { prevalente }(3 / 8) \\
\text { Manejo intrahospitalario } \\
\text { básico }(3 / 6)\end{array}$ \\
\hline Bioética & $\begin{array}{l}\text { Límites terapéuticos } \\
\text { frente a ensañamiento } \\
\text { terapéutico }(3 / 6) \\
\text { Principios básicos }(2 / 6)\end{array}$ & Honestidad (2/6) & $\begin{array}{l}\text { Límites terapéuticos frente } \\
\text { a encarnizamiento } \\
\text { terapéutico }(2 / 8) \\
\text { Comunicar malas noticias }(2 / 8)\end{array}$ \\
\hline $\begin{array}{l}\text { Comunicación } \\
\text { efectiva }\end{array}$ & $\begin{array}{l}\text { Relación M-P-F (5/6) } \\
\text { Cambio en el enfoque } \\
\text { paternalista de } \\
\text { la atención }(5 / 6) \\
\text { Empatía con los padres (4/6) } \\
\text { Credibilidad (4/6) }\end{array}$ & Relación M-P-F (4/6) & $\begin{array}{l}\text { Relación M-P-F (3/8) } \\
\text { Cambio en el enfoque } \\
\text { paternalista de la atención (3/8) } \\
\text { Importancia del lenguaje médico } \\
\text { en la comunicación (2/8) }\end{array}$ \\
\hline Gestión & $\begin{array}{l}\text { Nociones básicas (4/6) } \\
\text { Salud pública }(3 / 6) \\
\text { Trabajo en equipo (3/6) } \\
\text { Gestión de recursos }(3 / 6)\end{array}$ & $\begin{array}{l}\text { Nociones básicas }(2 / 6) \\
\text { Salud pública }(2 / 6) \\
\text { Trabajo en equipo }(2 / 6)\end{array}$ & $\begin{array}{l}\text { Salud pública (3/8) } \\
\text { Trabajo en equipo (2/8) } \\
\text { Herramientas de } \\
\text { administración (5/8) } \\
\text { Formulación y evaluación } \\
\text { de proyectos (2/8) }\end{array}$ \\
\hline $\begin{array}{l}\text { Análisis crítico } \\
\text { de datos, MBE }\end{array}$ & $\begin{array}{l}\text { Aplicación en el ejercicio } \\
\text { diario de la pediatría }(4 / 6)\end{array}$ & $\begin{array}{l}\text { Aplicación en el ejercicio } \\
\text { diario de la pediatría (2/6) } \\
\text { Búsqueda y selección } \\
\text { de la información (3/6) } \\
\text { Investigación }(2 / 6) \\
\text { Actualización permanente (2/6) }\end{array}$ & $\begin{array}{l}\text { Aplicación en el ejercicio } \\
\text { diario de la pediatría } \\
\text { Búsqueda de la información (3/8) } \\
\text { Lectura crítica (4/8) }\end{array}$ \\
\hline Docencia & Herramientas básicas (3/6) & Herramientas básicas (4/6) & Herramientas básicas (4/8) \\
\hline
\end{tabular}

'La integralidad en la atención del paciente es mucho más importante que el conocimiento técnico, que igualmente se puede adquirir'. (P1)

Se destacó el seguimiento de los niños mediante 'clínicas de continuidad' como herramienta de perfeccionamiento clínico; los líderes se refirieron a la necesidad de formar pediatras capacitados para asumir el rol de médicos de cabecera, tanto de niños sanos como de portadores de alguna enfermedad crónica.

‘..el pediatra ahí debería asumir el rol de cabecera, de confianza de la familia. Algo que no les enseñamos.' (E1)

Se recalcó la importancia de las competencias clínicas relacionadas con un adecuado diagnóstico, el tratamiento de la patología prevalente y la 
derivación oportuna, en el contexto de una actitud de actualización permanente.

\section{Competencias en bioética}

Las competencias en bioética, consideradas básicas en la formación de pediatría, fueron referidas espontáneamente por los entrevistados con mayor experiencia y mencionadas después de una reflexión más dirigida por los residentes en formación. Hubo un acuerdo respecto a la carencia en la formación actual.

'No tenemos ni siquiera lo básico.' (R5)

'Es uno de los temas más ausentes en nuestra formación.' (R4)

'Tengo dudas de cómo y cuánto visitan los residentes en la actualidad.' (E6)

Se consideró fundamental integrar los conceptos de forma transversal durante toda la duración del PFEP.

'Del comienzo al final del PFEP hay que abordar de forma permanente el tema de la bioética, debería ser un tema transversal.' (P2)

'Debe ser incorporada a las actividades diarias del residente, como parte de la formación integral.' (E6)

Hubo acuerdo respecto a que las discusiones éticas dependen de oportunidades frente a ciertas situaciones clínicas y la falta de docentes entrenados y dispuestos a guiar el aprendizaje significativo.

'Aprendemos más por conversaciones informales con algunos docentes que son modelos.' (R4)

'Lo fui aprendiendo de manera indirecta, pero no es parte del programa de posgrado.' (P5)

'En el PFEP tenemos discusiones aisladas en torno a los casos más complicados.' (P6)

'Depende de las iniciativas del docente.' (E5)

En opinión de los líderes, la falta de formación en bioética no sería responsabilidad exclusiva de los docentes, sino que además existiría una falta de interés y motivación de los residentes. Se destacó la diferencia de interés, en desmedro de los residentes licenciados del antiguo currículo de pregrado de medicina frente al nuevo.

'Hay dilemas éticos todos los días frente a cada paciente, pero parece que a los residentes no les interesa.' (E1)

\section{Competencias en comunicación efectiva en la relación médico-paciente}

La incorporación de la 'nueva' relación médicopaciente-familia, informada, horizontal, ya no paternalista, en el ejercicio de la pediatría, fue un aspecto muy destacado. No obstante, hubo acuerdo respecto al déficit en su formación y a la falta de docentes modelo, que enseñen a través del ejemplo.

'Vamos directamente a la enfermedad, y se nos olvida el ser humano que está al otro lado, se va perdiendo la sensibilidad, probablemente porque no se refuerza. Se refuerza sólo el conocimiento.' (R6)

'Estamos acostumbrados a atender pacientes con una actitud absolutamente pasiva, contemplativa. Cuando los papás son un poco más interactivos nos sentimos amenazados.' (R4)

'La gran mayoría de los residentes probablemente esté subentrenado, y eso se adquiere posteriormente.' (E8)

'No tenemos docentes que se desenvuelvan en esta área.' (E3)

\section{Competencias en gestión}

Unánimemente se reconoció la carencia de formación en gestión.

'Tema absolutamente desconocido.' (R4)

'No me siento competente en aspectos de gestión.' (P4)

'De gestión y administración no entregamos nada. Otros miembros del equipo de salud tienen más formación que los médicos.' (E5)

'Sin duda deberíamos entregarles más elementos sistematizados y la oportunidad para ejercitarlos.' (E6)

Se consideró relevante que los residentes adquirieran una formación básica en gestión, competencia vista como esencial para el enfrentamiento de los nuevos desafíos laborales; al respecto, los residentes con experiencia laboral previa, principalmente como generales de zona, tendrían una ventaja sobre los recién licenciados.

'Las ofertas laborales para actividades clínicas ya están prácticamente cerradas, al menos en Santiago, mientras que éstas van a ser opciones laborales reales y concretas.' ( $R 4)$

'Cada vez más, el médico necesita aplicar más conocimientos de gestión en su ejercicio diario, al menos conceptos generales.' (E8)

Entre las competencias específicas de gestión, se destacó la salud pública y el trabajo en equipo.

'En el PFEP se da menos la relación con el equipo de salud, son estamentos muy separados, no hay comunicación con el equipo, es como una isla.' (R2) 
'Deberíamos tener conocimientos y al menos una opinión sobre los planes de salud en la esfera pediátrica.' (P6)

Los líderes enfatizaron el rol del pediatra como líder de opinión a nivel público y de equipos de salud.

Pese a la indiscutible relevancia de la adquisición de competencias en gestión, algunos líderes opinaron que durante el PFEP se deberían entregar las capacidades básicas, con la opción de profundización para aquellos que manifiesten mayor interés, especialmente durante la educación continua.

'El PFEP debe dar las herramientas generales, luego la formación continua debe ir orientada hacia su interés y sus necesidades.' (E8)

\section{Competencias en medicina basada en la evidencia}

Los entrevistados consideraron imprescindible la adquisición de competencias en MBE y análisis crítico de información. Hubo coincidencia de que pese a que hay una formación teórica excelente, faltaría su práctica en la atención de los pacientes, debido a ausencia de docentes que la apliquen. Esto podría ser explicado por la falta de tiempo, falta de interés y por la resistencia al cambio de los académicos de mayor edad; en opinión de los licenciados, que fueron más autocríticos, esto se debería también a una falta de motivación de los residentes frente a la lectura crítica y enfrentamiento analítico de los pacientes; en este punto los líderes fueron aún más enfáticos y refirieron que los residentes buscarían la 'receta'.

'Tenemos una formación superbuena pero lamentablemente, ya sea por falta de facilidades o de interés, no la aplicamos diariamente, y si no se aplica se pierde.' (R1)

'Laformación en MBE es deficiente, ésta no se aplica por falta de tiempo y distintas prioridades.' (R2)

'Uno siempre encuentra que en el PFEP perdió el tiempo, que no sacó todo el provecho.' (P3)

'El problema de los docentes más viejos es que no lo saben y chocan con eso.' (E5)

'Falta la motivación de preguntarse el porqué, de justificarlo personalmente, les falta la mirada crítica, una actitud madura y definir su propia opinión y no aceptar la «receta».' (E1)

'No veo en los residentes una preocupación por estudiar, por tener su opinión. Repiten lo que le dicen los docentes; deberían cuestionar lo que se está haciendo, tener un interés por aprender.' (E3)

\section{Competencias en docencia}

Líderes, residentes y licenciados coincidieron en la importancia de adquirir herramientas básicas en docencia, competencia inherente al rol del médico.

'El médico, por su responsabilidad social, siempre va a ser un educador.' (R5)

'Compartir el conocimiento es parte del trabajo médico, ya sea con los semejantes, estudiantes, alumnos, pacientes.' (P6)

'El médico es un docente si cumple su labor integral de médico' (P1)

Se reconoció la carencia de herramientas de docencia durante el PFEP y se propuso que ésta sea una opción electiva durante la formación de pediatras. Se recalcó la importancia de docentes modelo en la formación de los residentes.

'No tenemos modelos que nos enseñen a enseñar.' (R4)

'Lo aprendemos como parte de un currículo informal, por docentes modelo.' (P6)

'El hospital tiene la gran gracia de tener un grupo de docentes que ejerce la docencia con el ejemplo, esto estimula a desarrollar la docencia' (E3)

\section{Discusión}

El PFEP debe responder a las necesidades futuras de la salud y las políticas del país, y modernizarse de acuerdo con las evidencias de la educación en ciencias de la salud $[19,20]$. En este contexto, además de la capacitación clínica, que es el núcleo de las especialidades médicas, y, en coincidencia, el aspecto más desarrollado según los entrevistados, es necesaria la incorporación formal e intencional de CT.

El mayor desafío, según distintos autores, radica en la implementación de un currículo basado en competencias [21]. Si consideramos que la clave para la consolidación de competencias está en su puesta en práctica [22], es necesario desarrollar programas orientados hacia las competencias, con uso de metodología docente innovadora [23], escenarios clínicos apropiados con docentes entrenados e imbuidos culturalmente en este modelo [24], que lo apliquen en el ejercicio diario y guíen el aprendizaje significativo. En este paradigma, debemos considerar, además, la responsabilidad de los residentes, quienes deben mostrar 
una visión amplia y una actitud proactiva en su proceso de enseñanza-aprendizaje.

Se podría plantear, entonces, que para lograr la adquisición de CT hace falta un cambio cultural en quienes planifican los programas, quienes lo enseñan y en los educadores. No obstante, los cambios culturales son lentos, y probablemente éstos aún no se han incorporado en el 'ser y actuar' de los académicos [22].

No deja de llamar la atención la gran relevancia que dan los entrevistados al rol de los docentes como modelos, especialmente para el aprendizaje de competencias tan fundamentales y arraigadas en el núcleo de los valores de las personas, como la bioética, la comunicación efectiva y la MBE.

Diversos estudios refieren que, no obstante, los valores éticos se forman en etapas precoces de la vida y podrían ser moldeados a través de ejemplos docentes en el currículo oculto; este aspecto, ampliamente analizado en publicaciones anglosajonas [25-27], coincide con la opinión de los entrevistados.

Se destacó la 'nueva' relación médico-pacientefamilia, informada, horizontal y ya no paternalista. Estudios realizados en nuestro país revelan que los médicos, principalmente los de mayor edad, mostrarían una percepción negativa del cambio en el modelo de relación [28], lo que sin duda debe reflejarse en las actividades docentes asistenciales.

Hubo consenso entre los líderes, respecto a la diferencia de formación entre los residentes del antiguo currículo de medicina [29] en comparación con los del nuevo, en cuanto a competencias relacionadas con bioética y comunicación médico-paciente; asimismo, un estudio reciente publicó la autoevaluación de los recién licenciados, quienes refirieron una adecuada capacitación en dichos aspectos [30]. Probablemente éste sea el comienzo del cambio cultural esperado para las generaciones de profesionales futuras.

Se discrepó respecto a si las competencias de gestión deberían ser consideradas como electivas o esenciales para los pediatras, pese a su relevancia para enfrentar el escenario laboral actual, en que la opción de trabajo clínico está saturada, fundamentalmente en las grandes urbes del país.

Hubo acuerdo sobre la importancia de que los pediatras estén capacitados para el trabajo en equipo y tengan formación en salud pública.

La oportunidad de entregar las competencias en docencia fue otro tema controvertido, pese a ser considerado como inherente al rol del médico. Internacionalmente se ha debatido la función docente del residente y la necesidad de preparación y evaluación de esta competencia durante los PFEP [31,32].

Con respecto a clínica, los entrevistados priorizaron la adquisición de capacidad resolutiva. Se reconoció unánimemente que la salud de los niños está influenciada por la familia, el entorno y el nivel socioeconómico [33,34]. Se destacó la importancia de aplicar el enfoque integral, biopsicosocial, en los distintos escenarios en los que se atiende a los niños, independientemente del motivo de consulta, asumiendo el rol de médicos de cabecera.

Se expresó la necesidad de reforzar las competencias en pediatría ambulatoria, pediatría del desarrollo y urgencias, coincidiendo con las recomendaciones formuladas por el Task Force on the Future of Pediatric Education (FOPE) $[35,36]$. Además, se propuso redirigir los PFEP hacia el enfoque integral y general del paciente hospitalizado, ya que los niños que actualmente se hospitalizan son de complejidad elevada y requieren de la visita de subespecialistas.

En conclusión, el núcleo de los PFEP es, con seguridad, clínico, por lo que las competencias clínicas entregadas deben ser acordes a la realidad epidemiológica y laboral [10], con mayor énfasis en la pediatría ambulatoria con enfoque biopsicosocial, urgencias pediátricas y manejo básico del paciente hospitalizado. No obstante, se evidenció que en el actual escenario de salud, las competencias clínicas por sí solas parecen no ser suficientes, y es necesaria la incorporación de nuevas capacidades en los programas de formación, en respuesta a los desafíos que enfrentará el pediatra del tercer milenio.

La debilidad de las CT en los actuales PFEP se debería a la ausencia de una formación intencionada y formal, pero también a la falta de motivación de los residentes.

Las principales CT mencionadas relacionan con la bioética, la relación médico-pacientefamilia, horizontal y ya no paternalista, la salud pública y el trabajo en equipo. La MBE debería aplicarse en el ejercicio diario para lograr su incorporación, más allá del fundamento teórico. La docencia, actividad inherente al ejercicio médico, podría ser ofrecida como un electivo, al igual que las competencias de gestión. 


\section{Bibliografía}

1. Hager P, Gonczi A. What is competence? Med Teach 1996; 18: 15-8.

2. De Miguel M. Modalidades de enseñanza centradas en el desarrollo de competencias: orientación para promover el cambio metodológico en el espacio europeo de educación superior. Oviedo: Ediciones de la Universidad de Oviedo; 2005.

3. Caraccio C, Wolfsthal s, Englander R, Ferentz K, Martin C. Shifting paradigms: from Flexner to competencies. Acad Med 2002; 77: 361-7.

4. Tuning Educational Structures in Europe. URL: http:// ec.europa.eu/education/policies/educ/tuning/tuning es.html. [20.05.2007].

5. Tuning educational structures in Europe. Informe final del Proyecto Piloto Fase 2. La contribución de las universidades al proceso de Bolonia. Pamplona: Universidad de Deusto; 2006.

6. Leung WC. Competency based medical training: review. BMJ 2002; 325: 693-6.

7. Patterson F, Ferguson E, Lane P, Farrell K, Martlew J, Wells A. A competency model for general practice: implications for selection, training, and development. $\mathrm{Br} \mathrm{J}$ Gen Pract 2000; 50: 188-93.

8. Accreditation Council for Graduate Medical Education. Outcomes Project 2001. URL: www.acgme.org/outcome [30.05.2008].

9. Perfil profesional médico-cirujano. Santiago: Universidad de Chile. Facultad de Medicina; 1998. p. 1-21.

10. Schonhaut L. ¿Es necesario replantear el programa de postítulo de pediatría con mayor énfasis a la pediatría ambulatoria?: perfil de competencias del residente de pediatría 2006. Universidad de Chile [tesis]. Santiago: Facultad de Medicina. Universidad de Chile; 2007.

11. Schonhaut L, Millán T, Hanne C. Formación de especialistas en pediatría y su adecuación a la realidad epidemiológica y laboral. Rev Chil Pediatr 2007; 78: 599-606.

12. Programas de Posgrado de Pediatría. Escuela de Posgrado. Facultad de Medicina. Universidad de Chile, 2006.

13. Patton M. Qualitative evaluation and research methods. Thousand Oaks, CA: Sage Publications; 1990.

14. Strauss A, Corbin J. Grounded theory methodology: an overview. In Denzin NK, Lincoln YS, eds. Handbook of qualitative research. Thousand Oaks, CA: Sage Publications; 1994. p. 273-85.

15. Instituto para la Educación Médica Internacional (IIME). Comité Central. Requisitos globales mínimos esenciales en educación médica. URL: http://wwwscielo.isciii. es/scielo.php?script $=$ sci_arttext \&pid $=$ S1575-18132 003000400003\&lng=pt\&nrm=iso. [02.06.2007].
16. Rosselot E, Chang N. Misión, visión y perfil profesional. Santiago: Universidad de Chile. Facultad de Medicina; 2003.

17. Unidad de Planificación de Educación Médica: evaluación de competencias de los estudiantes de la Carrera de medicina. Documento de trabajo UNEM; 2000.

18. Crabtree B, Miller W. Doing qualitative research. Thousand Oaks, CA: Sage Publications; 1992.

19. Schonhaut L. Los desafíos de la enseñanza de pediatría ambulatoria en el contexto del chile actual. Rev Chil Pediatr 2006; 77: 405-11.

20. Carraccio C, Englander R, Wolfsthal S, Martin C, Ferentz $\mathrm{K}$. Educating the pediatrician of the 21 st century: defining and implementing a competency-based system. Pediatrics 2004; 113: 252-8.

21. Batladen P, Leach D, Swing S, Dreyfus H, Dreyfus S General competencies and accreditation in graduate Medical Education. Health Affair 2002; 21: 103-11.

22. Ros-Martrat E. Las competencias profesionales adquiridas en medicina familiar y comunitaria: una mirada desde tutores y residentes. Educ Med 2004; 7: 29-35.

23. Baños JE, Pérez J. Cómo fomentar las competencias transversales en los estudios de ciencias de la salud: una propuesta de actividades. Educ Med 2005; 8: 40-9.

24. Sectish T, Zalneraitis E, Carraccio C, Behrman R. The state of pediatrics residency training: a period of transformation of graduate medical education. Pediatrics 2004; 114: 832-41.

25. Hafferty FW, Franks R. The hidden curriculum, ethics teaching, and the structure of medical education. Acad Med 1994; 69: 861-71.

26. Patenaude J, Niyonsenga T, Fafard D. Changes in students' moral development during medical school: a cohort study. JAMC 2003; 168: 840-4.

27. Hilton S. Medical professionalism: how can we encourage it in students? The Clinical Teacher 2004; 1: 69-73.

28. Bascuñán ML. Cambios en la relación médico-paciente y nivel de satisfacción de los médicos. Rev Med Chile 2005; 133: 11-6.

29. Herskovic P. La reforma curricular de la Escuela de Medicina de la Universidad de Chile. Rev Chil Pediatr 2005; 76: 9-11.

30. Millán T, Ercolano M, Pérez M, Fuentes C. Autoevaluación de habilidades clínicas básicas en médicos recién egresados de la Facultad de Medicina. Universidad de Chile. Rev Med Chile 2007; 135: 1479-86.

31. Morrison E, Hafler J. Yesterday a learner, today a teacher too: residents as teachers in 2000. Pediatrics 2000; 105: 238-41.

32. Zabar S, Hanley K, Stevens D, Kalet A, Schwartz M, Pearlman E, et al. Measuring the competence of residents as teachers. J Gen Intern Med 2004; 19: 530-3. 
33. American Academy of Pediatrics Task Force. Report on the future role of the pediatrician in the delivery of health care. Pediatrics 1991; 87: 401-9.

34. American Academy of Pediatrics, Committee on Psychosocial Aspects of Child and Family Health. The new morbidity revisited: a renewed commitment to the psychosocial aspects of pediatric care. Pediatrics 2001; 108: 1227-30.
35. Ambulatory Pediatric Association and Council on Medical Student Education in Pediatric. Core curricula in general pediatric. APA: 1994.

36. Scheiner AP. Guidelines for medical student education in community based pediatric office. American Academy of Pediatrics, Council on Pediatric Education and Subcommittee on Medical Student Curriculum. Pediatrics 1994; 93: 956-9. 